\title{
Intra-Articular Oxytetracycline for Synoviorthesis in the Treatment of Advanced Hemophilic Arthropathy
}

\begin{abstract}
Introduction: Hemophilia represents not a major case of consultation for the orthopedic surgeon; however, for the patient with hemophilic arthropathy, the condition may represent the major challenge regarding mobility and autonomy. Recurrent articular bleeding is a common cause of disability in the hemophilic patient, since joints suffering hemarthrosis follow an irreversible process of articular bleeding, joint erosion and degeneration, which reaches the end at premature arthrosis. In early stages, synovectomy is the treatment of choice to weaken the synovial tissue and prevent further bleeding.
\end{abstract}

Methods: We show the case of three adolescent patients undergoing chemical synovectomy with use of oxytetracycline. All three suffering recurrent knee hemarthrosis and one of them also with recurrent bleeding in dominant elbow. For a total of four knees and one elbow. Articular range of movement was assessed before treatment and during follow up in short and midterm. All treated knees showed grade III arthropathy before and after treatment. Elbow arthropathy was classified as grade II before and after treatment.

Results: Knee joints perimeter showed slight decrease since average preoperative perimeter was $31.25 \mathrm{~cm}$. and final perimeter was $28.37 \mathrm{~cm}$. In the elbow treated, decrease in perimeter decreased from $23 \mathrm{~cm}$ to $18 \mathrm{~cm}$. Articular bleeding episodes average 10.6 episodes per patient per year before treatment and decreased to 0.3 episodes per patient per year at final follow up.

Discussion: The aim of inducing fibrosis in synovial tissue by decreasing the amount of bleeding episodes is met with oxytetracycline sinoviortesis; however functional outcome of the patient does not improve, possibly due to start in an advanced stage of arthrosis (grade III - III).

Keywords: Hemophilia, Hemophilic Arthropathy, Synoviorthesis, Osteoarthritis, Oxytetracycline
Volume 7 Issue I - 2017

\author{
Douglas Colmenares-Bonilla,' Lauro \\ Vazquez-Ventura, ${ }^{2}$ Jesus Guerra-Jasso, ${ }^{3}$ \\ Loreette Ocampo-Perez ${ }^{3}$ \\ 'Pediatric Orthopaedic Surgeon, Head Couch in Pediatric \\ Orthopedics Speciality course, Hospital Regional de Alta \\ Especialidad del Bajio, Leon Guanajuato, Mexico \\ ${ }^{2}$ Orthopedic Resident, General Regional Hospital in Leon, \\ Mexico \\ ${ }^{3}$ Pediatric Orthopaedic Fellow, Hospital Regional de Alta
} Especialidad del Bajio, Mexico

Correspondence: Douglas Colmenares-Bonilla, Hospital Regional de Alta Especialidad del Bajio. Boulevard Milenio No. 130. Col. San Carlos la Roncha, C.P. 37660, Leon, Guanajuato, Mexico,Tel 477-267 2000,Email Douglas_cb@yahoo.com

Received: October 31, 2016 | Published: January 05, 2017

\section{Background}

Hemophilia, in the orthopedic background is not a major cause of consultation, however, for the hemophilic patient, arthropathy secondary to recurrent hemarthrosis, can mean the greatest challenge in mobility, pain, autonomy and quality of life. ${ }^{1,2}$ Similar results are expected for hemophilic families and/or caregivers through this progressive debilitant condition. ${ }^{3}$

The role of the orthopedic surgeon in hemophilia must be first preventive and later curative, thus osteoarthritis prevention is a must issue for all health personnel treating hemophilic patients, in order to decrease incidence and prevalence of hemophilic arthropathic damage and its aftermath. During pediatric age and development, it is common for hemophilic patient to sustain injuries in different magnitude, resulting in minor contusions with ecchymosis and edema, to highenergy trauma with serious lesions that may compromise life. ${ }^{2-4}$

But once hemophilic arthropathy is installed, the main purpose of medical or surgical treatment is focused in continuous rehabilitation, preserving muscle strength and joint range of motion. ${ }^{5-7}$ Moreover, very important after any episode of acute hemarthrosis, is try to prevent recurrence and thus accumulative joint damage, striving to delay osteoarthritis. ${ }^{8}$

Various studies associate more than two years of synovitis and low socioeconomic level as adverse factors to significantly increase the number of hemarthrosis episodes per year., ${ }^{910}$
Within the treatment of hemophilic arthropathy, synovectomy takes an important role as it seeks to reduce recurrences of joint bleeding through resection or ablation of subsynovial venous plexus. It can be performed by chemical or mechanical means, achieving synovial fibrosis and decreasing the frequency of hemarthrosis, which prolongs both integrity and function of the joint. ${ }^{2,5,11,12}$

The main risk of performing synovectomy by open or arthroscopic surgery, lies on trans and postoperative bleeding, as well as need of high amounts of specific coagulation factor, making surgery in an infeasible management to be conducted routinely in developing countries. ${ }^{5,7,13}$

Radioactive synoviorthesis is a relatively friendly treatment choice, that uses intraarticular radioactive isotopes to synovial ablation. Case description studies suggest to achieve encouraging results. Neverless, high cost and non-easy access to these drugs in developmental countries, leaves radioactive procedure in disadvantage compared to synoviorthesis with non-radioactive agents. ${ }^{14,15}$

In our regional context, the most viable procedure yields on chemical synoviorthesis, with intraarticular administration of antifibrinolytic and fibrosing substances, causing synovial atrophy and bleeding tissue eradication, thus preventing further hemarthrosis. ${ }^{5,6,12}$ Historically there have been described some substances for use in chemical synoviorthesis like talc, bleomycin, povidone iodine, sodium hydroxide etc. with encouraging results; however, the small number of patients and scarce clinical reports, turns them very risky to use. 
Intraarticular oxytetracycline for synoviortesis, have reported a clinical effectiveness about $80 \%$ in avoiding hemarthrosis recurrence when applied together with a physical therapy program ${ }^{5,12}$ and, as well as rifampicin, is the most accessible and cheap agent for this purpose. $^{6,12}$ Thus, intraarticular oxytetracycline currently represents our best option in management of early arthropathy, to stop repetitive bleeding.

It is a rule to start synoviorthesis as early as possible in order to avoid articular erosion and destruction, but when moderated to severe and advanced arthropathy is already present, there is no consensus about treatment of choice, since early arthrosis may lead to decrease quality of life in the individual, likewise early lose ambulatory status. ${ }^{16}$

\section{Methodology}

Chemical synoviorthesis treatment was performed in three patients with severe hemophilia and advanced arthropathy examined by the outpatient pediatric orthopedics clinic. All three of them had white joints, produced as result of hemophilic repetitive bleeding. Oxytetracycline was used intraarticular at dose of 400mg in large joints (knee) or $200 \mathrm{mg}$ in smaller joints (elbow) using $2 \%$ lidocaine as a vehicle for a total of $10 \mathrm{ml}$, at five weekly intervals for a total period of five weeks.

To assess the severity of chronic hemophilic arthropathy, Arnold and Hilgartner radiographic scale was used (Table 1), as well as the number of bleeding episodes in the previous 12 months. Functional assessment scale (Pediatric Outcomes Data Collection Instrument -PODCI) was filled before intervention and the end follow up. Clinical evaluation included diameter of the joint compared with the contralateral (if unaffected); range of movement and presence or absence of pain measured in VAS (visual analogue scale).

Table I Radiographic classification of hemophilia arthropaty

\begin{tabular}{|c|c|}
\hline Stage I & Increase in soft tissue without skeletal abnormalities. \\
\hline Stage II & $\begin{array}{l}\text { Hypertrophy and osteoporosis around the epiphysis. The joint } \\
\text { is stable. }\end{array}$ \\
\hline & Intra articular narrowing mild or moderate. Subchondral cysts. \\
\hline Stage III & $\begin{array}{l}\text { Widening of the intercondylar notch of the knee and elbow } \\
\text { sigmoid cavity. }\end{array}$ \\
\hline Stage IV & $\begin{array}{l}\text { Internal narrowing of the joint space with cartilage destruction. } \\
\text { Other bone changes very noticeable. }\end{array}$ \\
\hline Stage V & $\begin{array}{l}\text { Total loss of joint space with a fibrous ankylosis. Internal } \\
\text { incongruity of the joint structures with irregular epiphyseal } \\
\text { remarkable hyperplasia. }\end{array}$ \\
\hline
\end{tabular}

All patients had a regular program of physical therapy during and after periods of acute hemarthrosis and after every administration of oxytetracycline.

For drug administration, the specific anti-hemophilic factor were given intravenously 30 minutes prior to intra-articular oxytetracycline. Articular injection was followed by local cryotherapy for 20 minutes, and passive full-range of movement mobilization. All patients were discharged home 4 hours after drug administration once corroborated the general safe condition and absence of bleeding. In all cases, the indication for joint mobility and immediate weight bearing was given.

In case of acute hemarthrosis during treatment, the usual guidelines for this purpose were as follows:

I. Intravenous administration of the deficient factor (preferably within the first 2 hours of the traumatic event)

II. Immobilization of the joint for a maximum of 72 hours.

\section{Cryotherapy for periods of 20 minutes each $4 \mathrm{hrs}$ ( 5 days).}

IV. Early mobility and physical therapy (after $72 \mathrm{hrs}$ ).

Patients were followed for a minimum of 24 months, recording number of episodes of hemarthrosis in the treated joint, perimeter joint size, radiographic progression of hemophilic arthropathy, joint mobility and difference in perimeter of the treated joint with respect to the contra lateral side (in unaffected cases).

None of three patients received prophylactic hemophilic if no bleeding was observed due to lack of this support in our health system.

\section{Cases description}

\section{Patient I}

One Hispanic, 13 years old male, with confirmed diagnosis of severe hemophilia since he was 6 years, Joint symptoms in his right knee. He was suffering repetitive hemarthrosis since the last 4 years, accounting five events in the last 12 months.

Independently of right knee and secondary to indirect trauma, right elbow had limitation to mobility in flexion and extension, conserving pronation and supination. Hemarthrosis in right elbow accounted three episodes in last 12 months.

At first assessment, left elbow is observed with increased volume and perimeter $23 \mathrm{~cm}$, compared to $19 \mathrm{~cm}$. contralateral. The range of movement was 90 degrees of flexion with extensor lag of 50 degrees; useful mobility of only 40 degrees. For lower limbs, right knee had increased volume compared to the contralateral $(33 / 30 \mathrm{~cm})$. The range of movement in flexion of 90 degrees and extension lag of 60 degrees. He goes in wheelchair unable to walk since the previous five months.

Radiographs confirm a grade III hemophilic arthropathy in the right knee and grade II for the left elbow.

The protocol treatment was carried out with total time of 5 weeks at weekly interval applications, in both left elbow, and right knee.

As soon as joint bleeding episodes decreased, the patient initiated assisted walking and, together with physical therapy, regains ambulatory status and physical activities at home. He recovered independence for the toilet, transport and dress. The patient does not participate in any sport activity other than physical therapy.

In the third year of follow up the mobility of knee flexion is stated at 100 and extensor lag of 5 degrees.

At the end of a five-year follow-up, the functional outcome is acceptable, however it decreased right knee mobility, with flexion of 90 and extension of 40 degrees. Left elbow flexes 90 degrees and extends with a lag of 20 degrees. Radiographic arthropathy is grade II for the elbow and III in knee. The knee joint diameter is $32.5 \mathrm{~cm}$ (only $3 \mathrm{~cm}$ larger than the contralateral) and elbow $18 \mathrm{~cm} 2 \mathrm{~cm}$ far to the right. At five-years follow up, he present only one episode of right shoulder and knee hemarthrosis, own to height drop two months before the last appointment.

\section{Patient 2}

A male of 7 years, diagnosed with severe hemophilia at one year old. Hemarthrosis repetitive in his right knee for 3 years, with four episodes in last year. Last two hemarthrosis with minimal trauma. He maintained walking ability with right knee flexion and bending pelvis. Orthopedic reference was for lumbar pain since no other option for knee was given elsewhere. Clinical exam showed right 
knee to reach 85 degrees in flexion and 30 degrees in extension lag. Articular perimeter $37 \mathrm{~cm}(5 \mathrm{~cm}$ greater than the contralateral). Walking capable for few steps assisted on crutches in semi flexed right knee and shortening of $25 \mathrm{~mm}$. Radiographic classification as grade III arthropathy (Figure 1). Both physical therapy program and synoviorthesis help the patient to improve general quality of life and adapt himself to more independent life. Number of bleeding episodes decreased to one "slight" hemarthrosis in three years after treatment.

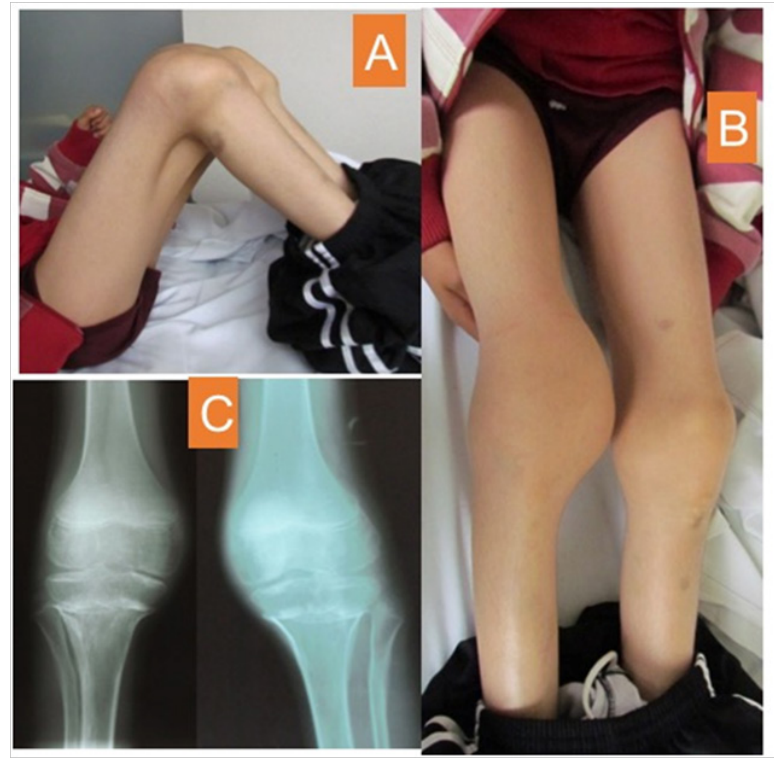

Figure I Inicial status of second patient. Seven years old male with acute insidious hemarthrosis of right knee.

A. Maximal passive flexion before treatment. B. Articular volumen increase incapable of complete extension. C. Radiographical changes corresponding to arthropaty grade III

After intraarticular treatment with oxytetracycline, a mobility of 90 degrees and flexion extension is achieved with a lag of 30 degrees at two years of follow-up.

At the end of four years, the patient is seen with semi-flexed knee. Mobility in extension lag of 10 degrees, flexion in range of movement of only 10 degrees. The $33 \mathrm{~cm}$ in diameter knee was not painful and only $2 \mathrm{~cm}$ larger than contralateral (Figure 2). At this point, he only manifested a new event in hemarthrosis of the left knee, three years after the synoviorthesis with no recurrence of bleeding.

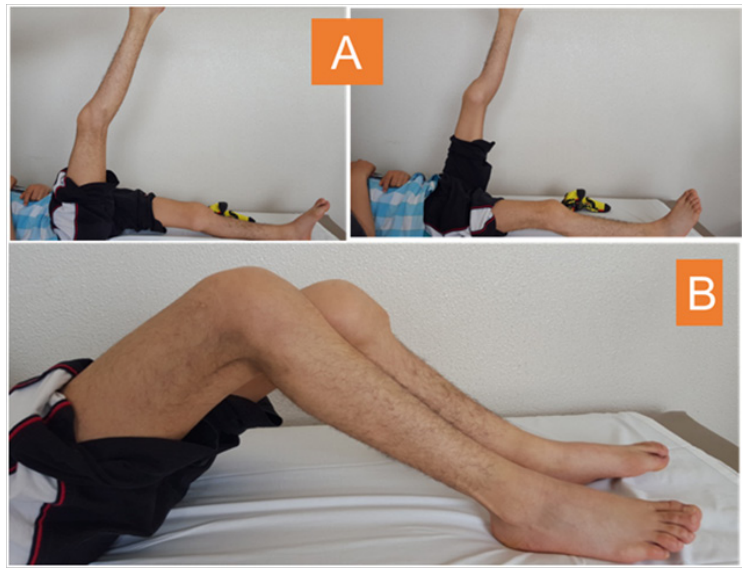

Figure 2 Final result after four years from treatment. A.Active extension and muscular status for both knees. B. Maximal passive flexion of knees. No pain was evocated wit this maneuvers.
This knee flexion noted painless mobility, but worsened to grade IV of hemophilic arthropathy on $\mathrm{X}$ rays (Figure 3 ).

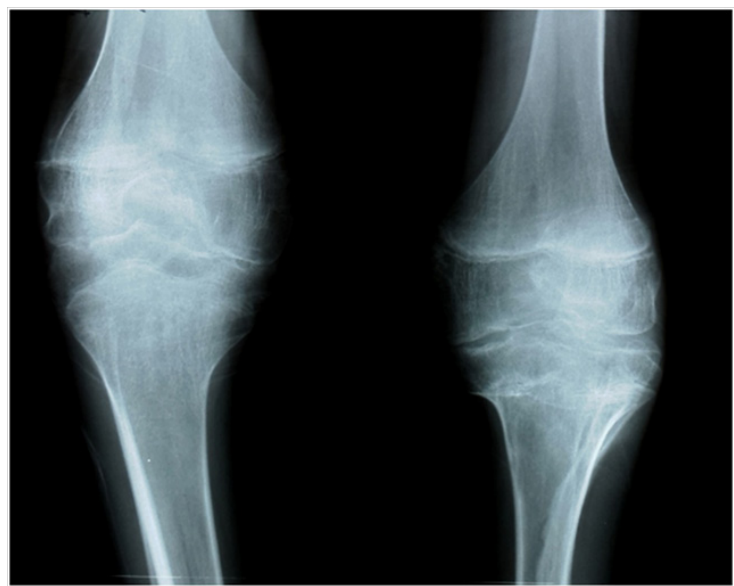

Figure 3 Final follow up for second patient, worsen radiographical imagen to be classified as grade III arthropathy four years after synoviorthesis.

\section{Patient 3}

Male 9 years, diagnosis of severe hemophilia since he was one year old. To his first orthopedic exploration arrived in a wheelchair, unable to walk since the past 3 months. He had history of repeated episodes of hemarthrosis in both knees nine times in the last 12 months. For bleeding episodes, he had received ice; cast and local physical rehab (mobilization) at home, being so frequent those episodes passed unattended for any physician.

The knees' diameter was 29/26 centimeters, flexion 120 and 90 degrees and extensor lag of 10 and 40 degrees. The radiographic classification for arthropathy grade III bilateral.

This patient after intraarticular treatment could walk on crutches since the fourth week been aided with physical therapy. After two years follow-up he showed mobility in flexion (120/120) and extension with lag of 10 and 35 degrees. The joint diameter 24 and $23 \mathrm{~cm}$ walking independently crutch assisted semi flexed knees.

At the end of follow-up for 38 months, both knees flexed 90 and 100 degrees, and lag extension of 25 and 20 degrees. He continues with an independent ambulation. Report of a single episode of hemarthrosis on right knee after two years of treatment.

Radiographs confirmed stage III of hemophilic arthropathy.

\section{Results}

It is to be noted that in all three patients the aim of abolishing the synovium with a use of oxytetracycline and decrease the number of joint hemorrhages per year is met, however the functional outcome is poor, because the limitation for the movement is the rule. The preexisting joint damage by previous bleeding is responsible for reducing the functional joint range of movement at the final follow up.

Chemical synoviorthesis help these patients to delay joint replacement surgery, by preserving ambulatory status and allow them to diminish another bleeding source and avoid risk related comorbidities.

This treatment is not intended for revert articular damage, but it is for delay the need of other procedures and help the patient maintain ambulatory status as much as possible, restoring so far autonomy and self-esteem. 
Worthy of mention is that in present study, range of movement at two year follow up is greater than initial, but decreases again after the fourth year. All range of movement caused no pain in last follow up.

\section{Discussion}

Compared with previous studies in patients with hemophilic arthropathy, it is suggested that the use of synoviorthesis with oxytetracycline hydrochloride is useful, offering fewer episodes of hemarthrosis and delayed joint degeneration, preserving mobility and better quality of life. It is a relatively simple procedure to be performed not only in specialized centers, but also in small health faculties.

The chemical synoviorthesis should start early to avoid the continued deterioration of articular patients due to recurrent bleeding.

The main weakness of our study is the small number of patients; however, they form a small sample of the racial characteristics of our population with advanced joint damage.

The early onset of synoviorthesis would avoid complications, improve the quality of life of hemophilic patient and should slow osteoarthritis.

It is necessary to conduct studies with more patients and longer follow-up to determine the use of these drugs, stablish rules for prevention and early treatment for acute and chronic arthropathy, which would increase quality of life and reduce costs of complications for the patient and health systems.

\section{Conclusion}

The most severe cause of disability in hemophilic patients is joint involvement by constant clinical and subclinical bleeding, occurring in up to $83 \%$ of patients. As a disease of low prevalence, it is common that the clinician not familiar with management in acute or recurrent stage of hemarthrosis stablish a sub optimal treatment, which allows rapid evolution of natural history towards early osteoarthritis. The synoviorthesis is a relatively safe technique that gives good results in patients with hemophilic arthropathy, even in advanced stages. Although it is imperative start this treatment in early stages of articular disease, even despite late start treatment, the benefit obtained is decrease number of bleeding episodes, which may delay osteoarthritis.

The best prognosis has been described in joints within stage I and II of the Arnold-Hilgartner scale, since arthritic changes are not yet observed and synovial destruction may limit the episodes of hemarthrosis. In present study we show that patients with stage III, have favorable results in reducing episodes of hemarthrosis (less than one per year after treatment), thus limiting blood-related chronic joint damage, which must lead to delay radiographic degenerative change. Range of movement and radiographic change was not possible may due to advanced previous joint status.

\section{Acknowledgments}

None.

\section{Conflicts of interest}

None.

\section{References}

1. Nijdam A, Foppen W, van der Schouw YT, et al. Long-term effects of joint bleeding before starting prophylaxis in severe haemophilia. Haemophilia. 2016;22(6):852-858.

2. Rodriguez-Merchan EC, Goddard NJ. Chronic haemophilic synovitis. In: Rodriguez-Merchan EC, et al. (Eds.), Musculoskeletal Aspects of Haemophilia. Oxford, Blackwell Science Ltd, UK. 2000;p.43-49.

3. Colmenares-Bonilla D, Ortiz-Agapito F. Impacto de los padecimientos ortopedicos pediatricos en la calidad de vida de la familia del paciente. Evid Med Invest Salud. 2015;8(3):117-121.

4. Perez JG. Patologías musculoesqueléticas en la hemofilia y tratamiento fisioterápico. Rev Biocienc. 2004;2:1-13

5. Fernandez-Palazzi F. Treatment of acute and chronic synovitis by nonsurgical means. Hemophilia. 1998;4(4):518-523.

6. Fernandez-Palazzi F, Rivas S, Viso R, etal. Synovectomy with rifampicine in haemophilic haemarthrosis. Haemophilia. 2000;6(5):562-565.

7. Rodriguez-Merchan EC. The Haemophilic Joints New Perspectives. Blackwell Publishing Ltd, USA. 2007;pp. 216.

8. Paez J, Hennessey R, Amador A. Efecto de la oxitetraciclina sobre el cartílago articular. Rev Col de Or Tra. 2006;20(3):48-54

9. Stein H, Duthie RB. The pathogenesis of chronic haemophilic arthropathy. J Bone Joint Surg Br. 1981;63(4):601-609.

10. Soroa V, Galatro G, Giannone C. Radiosinovectomias o radiosinoviortesis en hemofilia. Rev Asoc Argent Ortop Traumatol. 2005;70(1):68-81.

11. Roosendaal G, Vianen ME, Wenting MJ, et al. Iron deposits and catabolic properties of synovial tissue from patients with haemophilia. $J$ Bone Joint Surg Br. 1998;80(3):540-545.

12. Radossi $P$, Baggio $R$, Petris $U$, et al. Intra-articular rifamycin in haemophilic arthropathy. Haemophilia. 2000;9(1):60-63.

13. García-Chávez J, Majluf-Cruz A. Hemophilia. Gac Med Mex. 2013;149(3):308-321.

14. NF Tepeneu, C Luca, M Sabau, et al. Chemical Synovectomy Of The Knee In Rabbits-Oxytetracycline versus Aetoxysklerol-A Experimental Study. Jurnalul Pediatrului. 2011;14(14):53-54.

15. Luis Andrade V, Jorge Yarinsueca G, María Pacheco A, et al. Artropatia hemofílica, reporte de 23 casos. Revista Peruana de Reumatologia. 1995;1(2):1-8.

16. Raffini L, Manno C. Modern management of haemophilic arthropathy. Br J Haematol. 2007;136(6):777-787. 